\title{
Diagnostic Services for Melanoma in Italy
}

\author{
Ignazio Stanganellia Paolo Ascierto ${ }^{b}$ Riccardo Bonoc ${ }^{c}$ Vincenzo De Giorgi ${ }^{d}$ \\ Nicola Pimpinellid ${ }^{d}$ Vanna Chiarion-Sileni ${ }^{\mathrm{e}}$ Giuseppe Palmieri ${ }^{f}$ \\ Maria Antonietta Pizzichettag ${ }^{9}$ Alessandro Testori ${ }^{\text {h }}$ \\ astituto Scientifico Romagnolo per lo Studio e la Cura dei Tumori, IRCCS IRST, Meldola, ${ }^{b}$ IRCCS National Cancer \\ Institute, Naples, ' Immacolata Dermatological Institute, IRCCS, Rome, d Dermatologic Clinic, University of Florence, \\ Florence, ${ }^{e}$ Veneto Institute of Oncology, Padua, ${ }^{f}$ Genetica Oncologica, CNR, Sassari, ${ }^{9}$ Centro di Riferimento \\ Oncologico, Aviano, and ${ }^{\mathrm{h}}$ Istituto Europeo di Oncologia, Milan, Italy
}

\section{Key Words}

Melanoma $\cdot$ Nevi $\cdot$ Diagnostic services $\cdot$ Skin cancer unit .

Pigmented skin lesions - Dermoscopy · Digital dermoscopy . Epiluminescence microscopy $\cdot$ Mole mapping

\begin{abstract}
Objective: To evaluate organizational structure and diagnostic procedures used by the Italian hospital network for identifying cutaneous melanoma. Methods: A nationwide survey of a representative sample of centers was conducted. Results: Diagnosis occurs mainly in ambulatory dermatology clinics (91\%). In all high-volume hospitals, clinical and dermoscopic examination is available at first consultation or as an additional service, compared to $89 \%$ of low-volume hospitals. Computer-assisted videodermoscopy is available in $75 \%$ of hospitals, with a statistically significant difference between high- and low-volume hospitals ( 86 vs. $62 \%$; $p<$ 0.001 ). First consultation is generally an integrated clinical/ dermoscopic evaluation ( $55 \%$ of high-volume centers vs. $47 \%$ of low-volume hospitals); digital evaluation is available for monitoring suspicious lesions and high-risk patients in $25 \%$ of high-volume centers versus $19 \%$ of low-volume centers. Conclusions: The organizational structure and diagnostic procedures in Italian hospitals are in line with modern
\end{abstract}

\section{KARGER}

E-Mail karger@karger.com

www.karger.com/drm diagnostic procedures for early diagnosis of melanoma. Dermatologists have a central role in managing diagnosis of primitive melanoma.

Copyright $\odot 2013$ S. Karger AG, Basel

\section{Introduction}

Total body examination is the basic screening method for secondary prevention of melanoma. The main clinical signs of melanoma are summarized by the $\mathrm{ABCDE}$ rule $[1,2]$ and the 'ugly duckling' rule [3]. The ugly duckling rule seems to be a useful integration of the ABCDE rule which is based on lesion morphology ( $\mathrm{A}=$ asymmetry of the lesion; $\mathrm{B}=$ borders of the lesion are irregular; $\mathrm{C}=$ color: usually melanomas have many varied colors; $\mathrm{D}=$ diameter $>6 \mathrm{~mm}$; $\mathrm{E}=$ evolution over time), although sensitivity is low in early melanomas. Clinical examination includes evaluation of patient-reported lesions and the surrounding skin under magnification and strong lighting. Dermoscopy has been used as an adjunct to clinical examination since the late 1980s [4].

Dermoscopy (also called epiluminescence microscopy, dermatoscopy or reflected light microscopy) is a noninvasive technique that permits evaluation of dark, par- 
Table 1. Diagnostic and therapeutic services provided by Italian hospitals grouped according to yearly melanoma diagnoses into high-volume $(>25)$ and low-volume $(\leq 25)$ centers

\begin{tabular}{lcll}
\hline \multirow{2}{*}{ Service } & \multicolumn{3}{c}{ Type of center } \\
\cline { 2 - 4 } & $\begin{array}{l}\text { high- } \\
\text { volume } \\
(\mathrm{n}=56)\end{array}$ & $\begin{array}{l}\text { low- } \\
\text { volume } \\
(\mathrm{n}=64)\end{array}$ & all \\
& 100 & 97 & 99 \\
& 96 & 93 & 94 \\
Clinical diagnosis & $100^{*}$ & 89 & 97 \\
Excisional biopsy & 100 & 94 & 88 \\
Clinical and instrumental diagnosis & 94 & 83 & 95 \\
Histology & 98 & 92 & 82 \\
Radical surgery based on T & 85 & 78 & 72 \\
Medical therapy & $91^{* *}$ & 56 & 53 \\
Lymphadenectomy & $63^{* *}$ & 44 & 43 \\
Radical surgery and SLN procedures & & & \\
Participation in multicenter research & & & \\
$\quad$ protocols & 51 & 36 & \\
Genetic marker consultation and & & & \\
$\quad$ studies & &
\end{tabular}

Data are expressed as percentages. SLN $=$ Sentinel lymph node. ${ }^{*} \mathrm{p}<0.001 ;{ }^{* *} \mathrm{p}=0.003$.

tially pigmented or achromic neoformations under strong magnification. It facilitates identification of microscopic and vascular structures as well as pigment distribution, and improves the diagnosis of melanocytic lesions (particularly subtle melanomas) and non-melanocytic lesions (e.g., carcinoma, seborrheic keratosis, dermatofibroma, angioma and angiokeratoma) $[4,5]$.

With the introduction of digital dermoscopy, diagnostic management and follow-up of high-risk patients has improved [6]. Digital dermoscopy (videodermoscopy) involves evaluation and storage of clinical and dermoscopic images of melanocytic lesions to monitor for geometric, chromatic or structural changes [7]. Short-term application of this method (3-6 months) is used to monitor atypical lesions that may be featureless or nevus-like melanomas, while longer periods ( $>6$ months) are used to map and monitor multiple atypical moles in patients at high risk of melanoma $[7,8]$. Integrating management of clinical and dermoscopic diagnosis provides higher diagnostic efficacy compared to either examination alone [9]. Skin cancer prevention can therefore be summarized in the following steps: (1) clinical examination, (2) dermoscopic examination and (3) digital monitoring. The order of these steps may vary.

The aim of this study was to assess the organization of the Italian diagnostic service for melanoma in light of important diagnostic advances in early diagnosis of melanoma and mole mapping, such as digital dermoscopy.

\section{Methods}

Briefly, a nationwide survey of clinicians responsible for the diagnosis, therapy or follow-up phases of melanoma care in Italian hospitals was conducted. Italian hospitals with $\geq 200$ beds $(n=285)$ were subdivided into 145 hospitals with $200-399$ beds and 140 hospitals with $\geq 400$ beds and a proportionally stratified random sample ( $n=120$ centers), stratified by number of beds and geographic distribution, was selected. Two or three clinicians were interviewed at each center, resulting in approximately 250 interviews and a predicted margin of error - $95 \%$ confidence level - of $7.7 \%$.

Based on the findings, centers were grouped by number of new melanoma diagnoses per year into low- and high-volume centers, around the median value of 25 . Variables were analyzed in the total sample/total Italian hospitals, and comparisons were made between high- and low-volume centers using Pearson's $\chi^{2}$ test and the zeta test at $95 \%$ confidence level. Detailed methods are presented elsewhere in this issue [10].

\section{Results}

\section{Diagnostic Network}

Nearly all Italian hospitals (99\%) perform clinical evaluation of melanoma and pigmented cutaneous lesions (table 1). Diagnostic services are provided mainly in the dermatology departments (91\%) (table 2). The analysis shows that all high-volume hospitals provide clinical and instrumental examination at the first or second consultation, whereas the diagnostic level in the low-volume hospitals is lower ( 100 vs. $89 \%, \mathrm{p}<0.001)$ (table 1$)$.

Overall, $65 \%$ of centers have a specific unit for early diagnosis of melanoma and pigmented lesions, with a prevalence in high-volume centers ( $78 \mathrm{vs.} 52 \%, \mathrm{p}<0.001$ ). The pigmented skin units (i.e., dermatology oncology clinics) are generally integrated into the dermatology service.

In contrast, $22 \%$ of high-volume hospitals and $48 \%$ of low-volume hospitals $(\mathrm{p}<0.001)$ have dedicated dermatology oncology clinics that do not provide dermoscopic evaluation at first consultation. Another relevant finding is the availability of digital videodermoscopy in $75 \%$ of centers, overall, with a prevalence in high-volume hospitals ( 86 vs. $62 \%, \mathrm{p}<0.001)$.

\section{Diagnostic Steps}

Table 3 provides a description of the diagnostic procedure used in Italian hospitals for evaluating pigmented 
Table 2. Organization of diagnostic services in Italian hospitals grouped according to yearly melanoma diagnoses into high-volume $(>25)$ and low-volume $(\leq 25)$ centers

\begin{tabular}{|c|c|c|c|}
\hline \multirow[t]{2}{*}{ Service } & \multicolumn{3}{|c|}{ Type of center } \\
\hline & $\begin{array}{l}\text { high- } \\
\text { volume } \\
(\mathrm{n}=56)\end{array}$ & $\begin{array}{l}\text { low- } \\
\text { volume } \\
(\mathrm{n}=64)\end{array}$ & $\begin{array}{l}\text { all } \\
(\mathrm{n}=120)\end{array}$ \\
\hline $\begin{array}{l}\text { Clinic for diagnosis of pigmented } \\
\text { skin lesions }\end{array}$ & 100 & 52 & 65 \\
\hline General dermatology clinic & 86 & 96 & 91 \\
\hline \multicolumn{4}{|l|}{ Dedicated dermatologic oncology clinic } \\
\hline $\begin{array}{l}\text { No dermoscopy available on } \\
\text { initial visit }\end{array}$ & $22^{*}$ & 48 & 30 \\
\hline Dermoscopy available on initial visit & $78^{*}$ & 52 & 66 \\
\hline $\begin{array}{l}\text { Cutaneous diagnosis and instrumental } \\
\text { imaging service }\end{array}$ & $86^{*}$ & 62 & 75 \\
\hline
\end{tabular}

Data are expressed as percentages.

$* \mathrm{p}<0.001$.

lesions. The basic instrumental equipment in most hospitals allows skin examinations at first consultation, which are mainly carried out as part of an integrated clinical-dermoscopic evaluation (55\% of high-volume hospitals versus $47 \%$ of low-volume hospitals). Digital monitoring for melanocytic lesions and management of highrisk patients is available in $25 \%$ of high-volume hospitals versus $19 \%$ of low-volume hospitals. Few centers provide only clinical evaluation at first consultation, and this is more often the case in low-volume hospitals (34 vs. $20 \%$, $\mathrm{p}=0.02)$.

\section{Discussion}

The organization and diagnostic procedures used in Italian hospitals are rational and in line with modern diagnostic procedures for early diagnosis of melanoma [1$3,9-12]$. Dermatologists play an essential role in the management of primitive melanoma diagnosis.

There appears to be more interest in dermoscopy and digital applications in Italy, compared to other countries. This is evident from the elevated number of Italian studies published [12], which has accelerated the widespread use of the technique in combination with traditional clinical observation. The overall diagnostic situation is satisfactory, although significant differences exist between low- and high-volume hospitals for a number of structural aspects (clinics for the diagnosis of pigmented le-
Table 3. Diagnostic procedures envisioned for patients on their first visit to an Italian hospital, grouped according to yearly melanoma diagnoses into high-volume $(>25)$ and low-volume $(\leq 25)$ centers

\begin{tabular}{llll}
\hline Service & \multicolumn{3}{l}{ Type of center } \\
\cline { 2 - 4 } & $\begin{array}{l}\text { high- } \\
\text { volume } \\
(\mathrm{n}=56)\end{array}$ & $\begin{array}{l}\text { low- } \\
\text { volume } \\
(\mathrm{n}=62)\end{array}$ & $(\mathrm{n}=118)$ \\
\hline General visit & $20^{*}$ & 34 & 27 \\
$\begin{array}{l}\text { General visit and manual dermoscopy } \\
\text { General visit, manual and digital } \\
\text { dermoscopy }\end{array}$ & 55 & 47 & 51 \\
\hline
\end{tabular}

Data are expressed as percentages.

$* \mathrm{p}<0.02$.

sions using dermoscopy at first consultation, availability of videodermoscopy) and/or management aspects such as scheduled follow-up and distribution of the type of patients. Clinical-instrumental diagnostics are carried out in $94 \%$ of centers.

A recent meta-analysis revealed that dermoscopy is superior to naked eye examination for diagnosing melanoma in diagnostic reference centers [12]. However, the diagnostic accuracy of dermoscopy is not $100 \%$, and the final diagnosis must be based on a comparison of dermoscopy findings with anamnesis and clinical findings, including macroscopic morphology according to $\mathrm{ABCDE}$ and/or the ugly duckling rule [1-3, 9-13]. In practical terms this means that combination of clinical evaluation and dermoscopy reduces the risk of falsenegative diagnoses in melanoma $[11,13]$ and reduces the possibility of false-positive cases referred to surgical excision, thus limiting the risk of removing pigmented lesions defined as suspicious on clinical examination but diagnosed as benign on histological examination [14]. In addition, combined clinical and dermoscopic examination is only slightly longer than clinical examination alone [15].

In several highly technological countries, dermoscopy is not routinely taught in residency programs $[16,17]$, but data show an adequate level of training in Italy. In addition to offering combined clinical and dermoscopy evaluations at first consultation, most centers provide also the possibility of adding digital evaluation for monitoring melanocytic lesions and managing high-risk patients.

Recently there have been important advances in methods for the diagnosis of melanoma and management of 
high-risk patients. It is hoped that this information will encourage implementation of integrated clinical and instrumental evaluation of pigmented and hypopigmented skin lesions, thereby increasing diagnostic accuracy and the effectiveness of management.

\section{Disclosure Statement}

The authors received no funding and report no conflict of interest.

\section{References}

1 Whited JD, Grichnik JM: The rational clinical examination. Does this patient have a mole or a melanoma? JAMA 1998;279:696-701.

-2 Abbasi NR, Shaw HM, Rigel DS, Friedman RJ, McCarthy WH, Osman I, Kopf AW, Polsky D: Early diagnosis of cutaneous melanoma: revisiting the ABCD criteria. JAMA 2004;292: 2771-2776.

3 Gachon J, Beaulieu P, Sei JF, Gouvernet J, Claudel JP, Lemaitre M, Richard MA, Grob JJ: First prospective study of the recognition process of melanoma in dermatological practice. Arch Dermatol 2005;141:434-438.

4 Steiner A, Pehamberger H, Wolff K: In vivo epiluminescence microscopy of pigmented skin lesions. II. Diagnosis of small pigmented skin lesions and early detection of malignant melanoma. J Am Acad Dermatol 1987;17: 584-591.

5 Argenziano G, Soyer HP, Chimenti S, Talamini R, Corona R, Sera F, et al: Dermoscopy of pigmented skin lesions: results of a consensus meeting via the Internet. J Am Acad Dermatol 2003;48:679-693.

6 Stanganelli I, Burroni M, Rafanelli S, Bucchi L: Intraobserver agreement in interpretation of digital epiluminescence microscopy. J Am Acad Dermatol 1995;33:584-589.

7 Menzies SW: Cutaneous melanoma: making a clinical diagnosis, present and future. Dermatol Ther 2006;19:32-39.
8 Argenziano G, Mordente I, Ferrara G, Sgambato A, Annese P, Zalaudek I: Dermoscopic monitoring of melanocytic skin lesions: clinical outcome and patient compliance vary according to follow-up protocols. Br J Dermatol 2008;159:331-336.

9 Stanganelli I, Serafini M, Bucchi L: A cancerregistry-assisted evaluation of the accuracy of digital epiluminescence microscopy associated with clinical examination of pigmented skin lesions. Dermatology 2000;200:11-16.

10 Mingozzi E, Fregosi S, Gandini S, Stanganelli I, Chiarion-Sileni V, Testori A: Melanoma Task Force (META) project in Italy: methodology. Dermatology 2013;226(suppl 1): $1-2$.

11 Pizzichetta MA, Stanganelli I, Bono R, Soyer HP, Magi S, Canzonieri V, Lanzanova G, Annessi G, Massone C, Cerroni L, Talamini R; Italian Melanoma Intergroup (IMI): Dermoscopic features of difficult melanoma. Dermatol Surg 2007;33:91-99.

12 Vestergaard ME, Macaskill P, Holt PE, Menzies SW: Dermoscopy compared with naked eye examination for the diagnosis of primary melanoma: a meta-analysis of studies performed in a clinical setting. Br J Dermatol 2008;159:669-676.
13 Bowling J, Argenziano G, Azenha A, Bandic J Bergman R, Blum A, et al: Dermoscopy key points: recommendations from the International Dermoscopy Society. Dermatology 2007;214:3-5.

14 Carli P, de Giorgi V, Chiarugi A, Nardini P, Weinstock MA, Crocetti E, Stante M, Giannotti B: Addition of dermoscopy to conventional naked-eye examination in melanoma screening: a randomized study. J Am Acad Dermatol 2004;50:683-689.

-15 Zalaudek I, Kittler H, Marghoob AA, Balato A, Blum A, Dalle S, Ferrara G, Fink-Puches R, Giorgio CM, Hofmann-Wellenhof R, Malvehy J, Moscarella E, Puig S, Scalvenzi M, Thomas L, Argenziano G: Time required for a complete skin examination with and without dermoscopy: a prospective, randomized multicenter study. Arch Dermatol 2008;144: 509-513.

16 Nehal KS, Oliveria SA, Marghoob AA, Christos PJ, Dusza SW, Tromberg JS, Halpern AC: Use of and beliefs about dermoscopy in the management of patients with pigmented lesions: a survey of dermatology residency programmes in the United States. Melanoma Res 2002;12:601-605.

17 Freiman A, Barzilai DA, Barankin B, Natsheh A, Shear NH: National appraisal of dermatology residency training: a Canadian study. Arch Dermatol 2005;141:1100-1104. 